\title{
Cryo-SiN - A New Substrate to Monitor Viral Mechanisms
}

\author{
Justin R. Tanner ${ }^{1}$, Madeline J. Dukes ${ }^{2}$, Linda Melanson ${ }^{3}$, Sarah M. McDonald ${ }^{1}$ and Deborah F. Kelly ${ }^{1}$ \\ 1. Virginia Tech Carilion Research Institute, Roanoke, VA 24016, USA. \\ 2. Applications Science, Protochips, Inc., Raleigh, NC, 97606, USA. \\ 3. Life Sciences Division, Gatan, Inc., Pleasanton, CA 94588, USA.
}

Amorphous carbon is the conventional support material used to prepare biological specimens for cryoelectron microscopy (EM). Micron-sized holes produced in thin carbon support films provide a transparent background, ideal for imaging weak-phase objects such as viral assemblies (reviewed by Taylor and Glaeser, 2008) [1]. One inherent limitation in the use of holey carbon films is beam-induced movement due to charging effects [2]. Alternatives support films have been developed recently to mitigate these factors. Among these new substrates are conductive materials composed of titaniumsilicon metal glass $\left(\mathrm{Ti}_{88} \mathrm{Si}_{12}\right)$ [3] and silicon carbide (cryomesh) [4]. Both of these materials exhibited less charging effects in images of frozen-hydrated specimens. This indicates the use of new materials could greatly improve our capacity to study biological structures and mechanisms beyond current practices.

The use of silicon nitride ( $\mathrm{SiN}$ ) microchips to prepare specimens for single particle analysis is widely attractive due to their consistent manufacture [5]. As the production of carbon support films may vary widely, substrates that are invariably flat with impermeable, transparent surfaces would be of great benefit to the EM community. In our present work, we utilize SiN support films as active visualization platforms to monitor key processes in the rotavirus lifecycle. Rotaviruses are important pediatric gastrointestinal pathogens that infect host cells by 1) uncoating their outer capsids proteins to produce double-layered particles (DLPs) and 2) transcribing their RNA genome within the nascent DLPs [6]. Understanding the mechanisms by which viruses infect and replicate is essential for designing new vaccines and pharmacological reagents to prevent viral-induced diseases. Here, we demonstrate that rotavirus uncoating and transcriptional processes can be recapitulated on $\mathrm{SiN}$ microchips and directly visualized by single particle EM. These reactions require incubation steps that can be performed easily on impermeable SiN surfaces, whereas conventional carbon support films proved inadequate. Thus, the microchips played an interactive role in hosting microscale biological reactions. The concept of utilizing support films as active devices builds upon our recent work to develop Affinity Capture technology $[7,8]$.

Our present work shows that viral specimens typically produced on graphitized carbon support films may now be produced on SiN microchips. Likewise, SiN chips provide an improved surface for capturing active viral assemblies using Ni-NTA lipid coatings (Figure 1). The microchips could be vitrified using "gentle-blot" conditions to make highly reproducible cryo-EM specimens. Thus, we have aptly named this material, Cryo-SiN. Our initial findings indicate that DLPs prepared on Cryo-SiN, exhibited greater contrast in comparison to those prepared on holey carbon films under the same conditions. As a result, we were able to calculate 3D reconstructions of rotavirus particles prepared on Cryo-SiN using a fraction of the images required for samples prepared on holey carbon films (Figure 2). Collectively, our findings suggest that SiN chips can serve as a versatile microscale bioreactor to monitor the mechanistic progression of the rotavirus lifecycle. We anticipate these devices will now permit us to study viral processes in greater detail and with more physiological relevance. 


\section{References:}

[1] K. A. Taylor and R. M.Glaeser, J. Struct. Biol. 163 (2008), pp. 214-223.

[2] Brilot, A. F, J. Z. Chen, A. Cheng, J. Pan, S. C. Harrison, C. S. Potter, B. Carragher, R. Hendersen and N. Grigorieff, J. Struct. Biol. 177 (2012), pp. 630-637.

[3] Rhinow, D. and W. Kuhlbrandt, Ultramicroscopy 108 (2008), pp. 98-705.

[4] Yoshioka, C., B. Carragher and C. S. Potter, Microsc. Microanal. 16 (2010), pp. 43-53.

[5] Ring, E. A., D. B. Peckys, M. J. Dukes, J. P. Baudoin and N. de Jonge, J. Microsc. 243 (2011), pp. 273-283

[6] Trask, S. D., S. M. McDonald and J. T.Patton, Nat. Rev. Microbiol. 10 (2012), pp. 165-177.

[7] Degen, K., M.Dukes, J. R. Tanner and D. F. Kelly, Rsc. Adv. 2 (2012), pp. 2408-2412.

[8] Gilmore, B. L., S. P.Showalter, M. J. Dukes, J. R. Tanner, A. C Demmert, S. M. McDonald and D. F. Kelly, Lab Chip 13 (2013), pp. 216-219

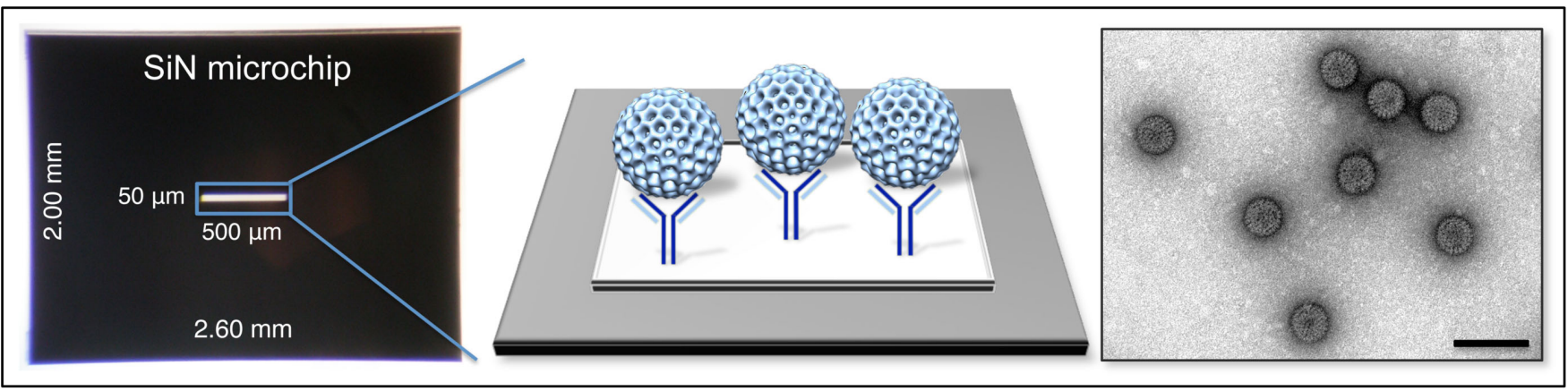

Figure 1. SiN microchips (left) containing 50-nm thick transparent windows were functionalized and decorated with protein $\mathrm{A} / \mathrm{IgG}$ antibodies to recruit viral complexes (middle). Negatively stained EM image (right) of rotavirus DLPs binding specifically to the SiN microchips. Scale bar is $150 \mathrm{~nm}$.
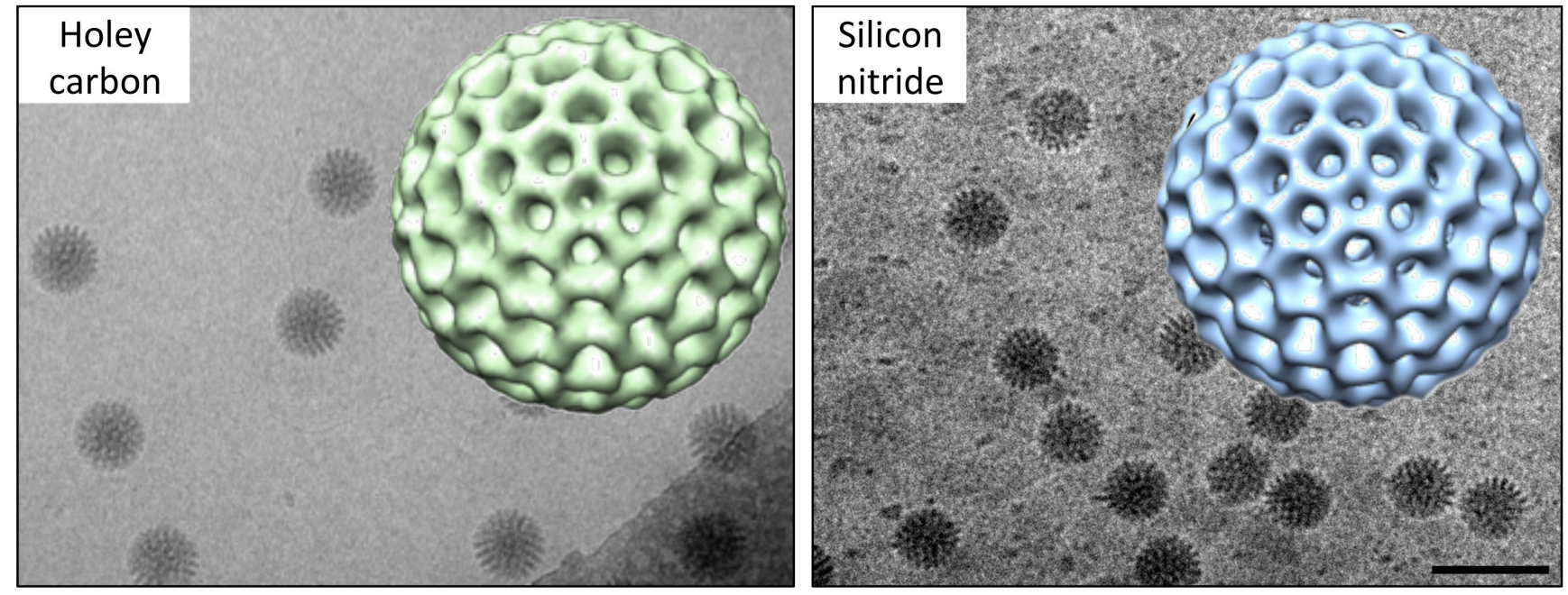

Figure 2. Images and 3D reconstructions (insets) of DLPs frozen on holey carbon grids (left) exhibited less features than those prepared on Cryo-SiN (right) under the same conditions. Molecular adaptors can be seen in the background of the specimens prepared on silicon nitride. Scale bar is $150 \mathrm{~nm}$. 